\title{
Distribution of Data Handling in Cloud Asset
}

\author{
Chetana Tukkoji, Boosi Shyamala, Archana S. Nadhan, Rashmi K.
}

\begin{abstract}
In Cloud computing, task scheduling is one of the technique of specifying and assigning job to assets that finish the job. It may be virtual computing elements like threads \& processors or data flows, which is planned on hardware resources like processors. The planning operation is performed by a scheduler. Schedulers are enabled various customers to properly communicate system funds or attain excellent service quality. Scheduling is essential for computing and the notion of planning allows multitasking computers with single CPU as inner portion of a computer system's execution model. Preference will be provided based on the requirements and goals of the user. Multiple computing parts comprise of many parallel applications while duties of execution are relied on other duties. We have studied few related articles in this paper, which is presented in the following section.
\end{abstract}

Keyword: Task Scheduling, Threads, Multi-tasking.

\section{I.INTRODUCTION}

Pei Yun Zhang et.al [1] has been suggested as a technique is centered on a two-stage approach is to improve job planning efficiency then minimize unreasonable job distribution in clusters. At first phase, a work classifier was used to identify assignments are dependent on historical planning information, driven by planning concept of a Bayes classifier. Certain amount of different kinds of virtual machines (VMs) are developed appropriately. This can save money during task scheduling to create VMs. Tasks are dynamically combined with concrete VMs in second phase. Therefore, dynamic algorithms for planning tasks are suggested. Experimental findings indicate that they can efficiently enhance the planning efficiency of the cloud and attain load handling of cloud assets are compared to current methods. Task planning is one of the difficult issues in cloud computing when considering deadlines and costs. Virtual machines (VMs) serve a crucial part in the planning of cloud tasks as a significant actuator. To fulfill the job deadlines, it is necessary to save time for generating VMs, planning time for tasks, and moment for execution. To minimize the expense of executing the job, assignments must be scheduled for execution on their appropriate VMs. To do this, a cloud task scheduling structure was suggested depending on two-stage approach. It generates VMs are based on historical planning information, thus reducing time for assignments are waiting for VMs to be created.

Revised Manuscript Received on February 05, 2020.

* Correspondence Author

Dr. Chetana Tukkoji, Assistant Professor, Department of CSE, GITAM School of Technology- Bengaluru campus, Bangalore, India.

Mrs. Boosi Shyamala, Assistant Professor, Department of CSE, GITAM School of Technology- Bengaluru campus, Bangalore, India.

Mrs. Archana S. Nadhan, Assistant Professor, Department of CSE, GITAM School of Technology- Bengaluru campus, Bangalore, India.

Ms. Rashmi K., Assistant Professor, Department of CSE, GITAM School of Technology- Bengaluru campus, Bangalore, India.

(C) The Authors. Published by Blue Eyes Intelligence Engineering and Sciences Publication (BEIESP). This is an open access article under the CC BY-NC-ND license (http://creativecommons.org/licenses/by-nc-nd/4.0/)
It dynamically combines duties with most appropriate VMs, thus reducing the expense of their execution. It reduces the waiting time of VMs for task scheduling under the premise of reaching deadline of task, thus minimizing the costs to be paid by users using VMs. The easily deployable algorithms are intended and demonstrated to enhance the planning and performance of cloud tasks are compared to traditional techniques.

ZhifengZhonget.al [2]: A Greedy Particle Swarm Optimization (G\&PSO) method has been implemented to fix the issue of planning tasks. A greedy algorithm to rapidly fix original particle valuation of algorithm for particle swarm analysis is found from cloud platform is based on virtual machine. The recorded test findings are indicated that the algorithm shows efficiency on each virtual machine, such as a quicker convergence rate, local and global query capacities, and controlled workload. The G\&PSO engine is enhanced the effectiveness and use of simulated machines are relative to traditional particle swarm optimization algorithm.

ChuanLinet.al [3] regarded two types of data shift planning issues: single-file transfer scheduling (SFTS) and multi-file transfer scheduling (MFTS), both are involved date restriction for file transition. A heuristic algorithm along with a precise SFTS-A algorithm was suggested to tackle SFTS issue. Compared to the precise algorithm, the heuristic algorithm requires less moment to run and has been prepared to tackle the SFTS issue effectively even if alternative may not be ideal, which simulation findings have properly demonstrated. For MFTS problem, they proposed a heuristic MFTS-H algorithm with dynamic reconfiguration scheme for multiple concurrent files to address transfer scheduling problem. The respective simulation findings show that in addition of delay and bandwidth usage, the proposed algorithm can effectively sustain various file transfer across various routes.

ZHAO Yong et.al [4] has been shown on many task computing (MTC) which is fresh category of computing paradigm in which the aggregate amount of assignments, computing quantities and information quantities can be big. With the trending technology like, cloud computing and the growth of big data, the efficient planning and execution of large-scale computing activities and the reasonable allocation of funds to assignments are becoming challenge. They have arrived to current task scheduling algorithm with asset feature choice to enhance both job execution and asset usage effectiveness, which can pick ideal node to perform a job according to its energy demands and fitness between the storage node and the job. Results of the experiment is indicated that the execution throughput and asset usage is improved significantly relative to other three algorithms and four planning frameworks.

The queuing model with exhaustive delivery was suggested by Chunling Cheng er.al [5] to model the job time table of a heterogeneous cloud computing scheme. 
Next, they evaluated the results of the job so journal moment and energy consumption nodes in various cloud computing scheme is depending on working era and idle interval in steady state. Subsequently, to decrease energy consumption, they suggested task scheduling algorithm is depending on comparable assignments. Results of the simulation indicate that the suggested algorithm can efficiently decrease the cloud computing system's power consumption while performing the job.

Nicholas H et.al [6]: a layout has been suggested that clearly addresses particular implementation features those are decoding dependencies, decoding deadlines and effects of distortion in various image streams (e.g. pixels, rows, slice associations, etc.). Cross-layer layout is one of the resource management control plane (RMCP), which controls planning and choice of processors across current apps. The RMCP exposes a priority-queuing model it will assess the flow of the scheme and estimate the complete video quality required for effective decoding assignments. They created a solid distortion-and delay-aware algorithm for the planning of audio transmissions using this model. This algorithm seeks to increase overall decoded audio frames the amount of video quality attained. They also proposed a selection scheme for the processor to reduce the delays experienced by the queued video packets. This intern reduces the number of decoding deadlines lost and increases general performance of decoded audio. They contrasted queuingtheoretical scheduling strategies with press agnostic scheduling strategies (i.e. earliest-deadline-first planning), which did not weigh the decoding deadlines and the effects of distortion together. Our findings show that considerable device efficiency improvements in the range of $4 \mathrm{~dB}$ PSNR ratios can be accomplished by straight contemplating the characteristics of the image application in the layout of a video decoding scheme.

HaitaoYuan et.al [7] has shown that cloud-based economies of scale attract an increasing amount of organizations and manufacturing firms to implement Apps in cloud datacenters (CDCs) and also provides facilities to the customers worldwide. The ambiguity of reaching duties leaves a major task for personal CDC to plan delay-bound projects costeffectively without surpassing their delay limitations. Unlike past research, HaitaoYuan et.al took up the cost minimization issue in hybrid clusters for personal CDC, where energy price of personal CDC and execution price of government clusters are indicated the temporal variety. They then suggested a temporal task scheduling algorithm (TTSA) to deliver all arriving duties efficiently to personal CDC and government servers. The cost minimization issue was designed as a blended integer linear program in each TTSA phase then fixed through a hybrid simulatedannealing particle-swarm optimization. The test findings showed that ideal or suboptimal planning approach is generated by TTSA can boost the throughput effectively then decrease the price of personal CDC while fulfilling the late limits of all activities are relative to the current techniques. Hui Zhao et.al [8]: concentrated on Map Reduce and it is a common cloud computing programming system for dealing with heavy computing tasks, example video transcoding. It divides the image (assignment) into various sections (subtasks) and transcodes them. Because of the complexity of video transcoding and also poor performance of the heterogeneous Map Reduce cluster, it was still challenge to schedule those part of tasks to reduce its total

transcoding time. They suggested in this article a predictive and locality-aware technique of planning tasks to parallel video transcoding over a heterogeneous Map Reduce cloud. Firstly, they analyzed technologies for video decoding/encoding and prediction of complexity of the transcoding segment that can provide a foundation for following scheduling. Secondly, they try to plan sub-tasks on devices containing input data was referred to as information location, to decrease large-scale data movement and information exchange in mapping phase. Third, they formulated the planning as an issue of Job Shop Scheduling (JSS) and suggested a heuristic prediction-based and locality aware task scheduling algorithm called PLTS. It incorporates the advantages of two heuristic scheduling algorithms are Max-Min and Min-Min to create group load balancing and to shorten the complete transcoding time. The test findings also demonstrated their algorithm's effectiveness.

Hongyan Cui et.al [9]: concentrated on cloud computing allowed huge network connectivity to a range of configurable computing assets for customer exchange. To execute duties and apps efficiently and reliably in a largescale, extremely heterogeneous setting, it must allocate resource adaptively. Cloud computation resource distribution is an NP-hard issue. In this document, we use a Markov-based technique to perform a reliability analysis of cloud services. We formulate the issue of cloud planning as a multi-objective issue of optimization with quality limitations, making pan and stream moment. In addition, we are proposing a Genetic Algorithm-based Chaotic Ant Swarm (GA-CAS) algorithm in which four drivers and natural selection are implemented to fix this multi-objective optimization issue. Results from simulation have shown that GA-CAS usually accelerates convergence and outperforms other meta-heuristic methods.

SérgioEsteveset.al [10]: suggested that data-intensive and long-lasting workflow Apps be progressively shipped to cloud computation devices. Present scheduling approaches are fail to deliver high resource efficiency while keeping computation costs low for dependency graphs, especially for continuous data processing workflows where the scheduler does not rationalize the impact new input data might have on the final output of the workflow. They launched fresh planning criteria, Quality-of-Data (QoD), which defines the information specifications capable of causing assignments in workflows to confront such a difficulty. A novel serviceoriented scheduler schedule for ongoing information handling workflows was suggested on the basis of the QoD concept, which was able to enforce QoD limitations and direct planning to achieve resource efficiency, general monitored results and job prioritization. To compare the benefits of our planning model with others, they created a cloud workflow assistant scheme where information was exchanged through cloud columnar database between assignments. Haitao Yuan et.al [11]: suggested cloud-based task scheduling and an increasing amount of businesses are deploying their apps in green data Centre's (GDCs) and providing facilities to worldwide user functions. An increasing amount of GDC suppliers are currently aiming to enhance their profit by exploiting green energy equipment and reducing consumption of brown energy. 
However, the temporary variability in income, electricity cost, and green energy in task wait boundaries leaves it difficult for GDC suppliers to accomplish profit maximization while fully ensuring that all accepted assignments are wait limitations. Unlike current research, a time aware task scheduling (TATS) method has been suggested to investigate the temporal variability and schedules all confessed assignments in GDC to fulfill their delay limits. Moreover, this article offers the task's mathematical modeling that a property delivery prices were denied. In each iteration, TATS solve the issue of defined profit maximization relying on simulated annealing by hybrid nonlinear plasma hot optimization. TATS can improve profit and throughput compared to several current scheduling algorithms without breaking the time limitations of all admitted tasks.

\section{II.FINE-GRAINED RESOURCE-AWARE SCHEDULING FOR MAPREDUCE}

Zhang et.al [12]: focused on Map Reduce as it has become a common model in latest years for data-intensive computing. By slowing down each task into a tiny chart and reducing duties and operating them on a big number of computers in conjunction, Map Reduce can considerably reduction the runtime of data-intensive employment. Despite latest attempts to design resource-efficient Map Reduce schedulers, however, current approaches that concentrate on task-level planning still provide sub-optimal job performance. This was because jobs during their lives can have extremely variable energy demands, which leaves it hard for schedulers at the task-level to efficiently use accessible funds to decrease the moment of execution of jobs. In order to tackle this restriction, they implemented PRISM, a fine-grained resource-awareness Map Reduce scheduler which separates duties into stages where each stage has a steady resource utilization to conducts phaselevel planning. They first proved the significance of phaselevel planning by using a wide range of Map Reduce employment to show the energy utilization variation during the lifespan of a job. They then submitted a phase-level scheduling algorithm that increases parallel execution and asset usage without ant straggle being introduced. PRISM offers elevated energy usage in a 16-node Hadoop array operating normal benchmarks and provides 1.3 changes in the runtime of work relative to the present Hadoop schedulers.

\section{III.TASK SCHEDULING IN THE CLOUD}

Xiaomin Zhu et.al [13]: suggested this proposal and energy conservation was a significant problem in cloud computing devices because it can deliver several significant advantages such as lowering operating costs, improving device efficiency to protect environment. Meanwhile, a strategy to power-conscious planning was a successful route to attain that objective. At the same moment, many real-time apps have been implemented in clusters, such as signal processing, scientific computing. Unfortunately, current energy-aware scheduling algorithms created for satellites are not tailored to real-time tasks and therefore lack the capacity to guarantee schedulable of the scheme. A novel approach for horizon planning architecture for real-time task scheduling in virtualized windows was suggested in this article to tackle this problem. Then evaluated a task-oriented model of energy consumption. They created a new energyaware planning method called EARH for real-time, aperiodic, autonomous assignments based on our planning design. The EARH uses an optimization strategy for rolling horizons and can also be expanded to include other energyaware scheduling algorithms. In addition, two asset scaling and scaling policies were suggested to create a healthy trade-off between the schedulability of the task and energy conservation. Extensive simulation studies injecting arbitrary synthetic assignments as well as assignments regarding the latest variant of Google's cloud trace records are performed to validate our EARH's supremacy by linking it to some baselines. The test findings indicated that EARH considerably increases other people's planning performance and were appropriate for managing real-time tasks in virtualized cloud.

Yang et.al [14] concentrated on Vehicular ad hoc networks (VANET), wireless device networks, Internet of Things (IoT), and portable device-to-device connections as various homogeneous fog networks where comparable terminals / things / devices / nodes share their neighborhood computing, interaction and memory assets to achieve greater device efficiency through effective collaborations. Simultaneously reducing delivery time and energy consumption in such networks for delay-sensitive and energy-constraint apps, such as virtual reality and internet $3 \mathrm{D}$ gaming on mobile devices, is highly desirable, but quite difficult. In this paper a cross-sectional analytical framework was established to formulate and investigate the balance between client disruption and energy consumption. An effective control parameter $\mathrm{V}$ is achieved to characterize their commercial connecting during vibrant task scheduling processes in cloud networks. A Delay Energy Balanced Tasking Scheduling (DEBTS), combined with estimation methods, has been proposed to minimize overall power usage while reducing average client delays and costs. DEBTS demonstrate the theoretical [O (1/V), O (V)] compromise between the twoeffectiveness metrics. Furthermore, extensive simulation results showed that DEBTS can offer much higher delay energy efficiency in task scheduling problems. DEBTS can, in particular, save \$26 and 29 cents more energy at a typical $\mathrm{V}$ rate of 4 to 114 . Simultaneously, the medium client duration is 29 cents and 32 cents lower than the traditional Random Timetable and Low-Business Timetable algorithms. In the light of the diversity of resources and cost efficiency, Christina Delimitrouet.al [15] suggested that the cloud suppliers are hosting a growing range of common apps. Most of these schemes have revealed a range of virtualized assets. As cases contain the same physical base, they argue on storage assets, e.g. the last spare, rendering them susceptible to co-programmed apps side-channel assaults. In this study, they demonstrated that by using information mining technology, an opponent in the cloud can determine the essence and features of co-programmed apps and adversely affect their efficiency through specific dispute injections. They have developed Bolt, a straightforward run-time that removes sensitivities for different kind of interference from co-programmed apps and utilizes this message to define the sort of apps using a number of information mine technologies and the precision of Bolt on a 39-server grid. 
Out of 108 casualty requests, Bolt properly recognized the sort and features of $81 \%$ and builds specific dispute messages to degrade its efficiency. The most common apps on EC2 were found with Bolt.

And highlighting these safety vulnerabilities in contemporary cloud equipment will encourage cloud suppliers to implement more primitive asset separation into their applications.

XingquanZuoet.al [16]: concentrated on Public Cloud job planning to provide customers with inadequate computer funds with Infrastructure as a Service (IaaS). IaaS achieves economies of scale through multiplexing and thus presents the task of planning assignments to satisfy peak demand while maintaining Quality of Service (QoS). Previous surveys suggested proactive buying of machines or cloud federation to solve this issue. The former is not financial, however, and the latter is hardly practicable for now. In this document, we suggest a structure for resource allocation in which an IaaS supplier can outsource their duties to external clouds (ECs) when their own funds are not adequate to satisfy the requirement. No official inter-cloud contract is required for this architecture for the cloud federation. The main problem is how customers ' duties can be allocated to enhance the IaaS provider's profit while ensuring QoS. This issue is developed as a model for integer programming (IP) and is fixed through a planning strategy based on selfadaptive learning particle swarm optimization (SLPSO).In SLPSO, in order to guarantee its variety and robustness, four updating techniques are used to adapt the velocity of each particle. Experiments demonstrate that, relative to conventional PSO, SLPSO can enhance the gain of a web provider by 0.25 percent -11.56 percent; and by 2.37 percent -16.71 percent for non-trivial volume issues relative to CPLEX in decent computing moment. Note to Practitioners IaaS has become a significant paradigm in cloud computation and many businesses offers such as Amazon EC2, IBM Smart Cloud Enterprise, and Microsoft Azure are available. IaaS suppliers are experiencing a major dilemma as to how to plan their assets to satisfy the requirement while guaranteeing the guaranteed QoS. We suggest a structure that allows an IaaS supplier to outsource their workloads to internal servers when their own funds are not enough. A SLPSO is used to plan inter-cloud funds efficiently. Compared to the web union, this structure is more practical because no company contract is needed to be achieved in time. The planning strategy is capable of finding the ideal or suboptimal distribution system of inner and external funds to enhance IaaS suppliers ' gain and can significantly enhance the planning solution's performance.

\section{IV.ADAPTIVE DISPATCHING OF TASKS IN THE CLOUD}

Lan Wang et.al [17] provided automatic cloud dispatching and the increasing use of cloud computing allowed dozens of thousands of apps to be consolidated in mutual infrastructure. Meeting the QoS demands of so many different apps in such shared resource settings has thus become a true task, particularly as apps ' features and workload vary extensively and may alter over moment. This article provided an experimental system capable of exploiting a range of internet QoS-conscious flexible job distribution systems and designing and comparing three such systems. These were an algorithm powered by

estimation that utilizes reinforcement learning, secondly a "sensitive" distribution engine that transfers duties to subsystems that are expected to provide a reduced response time, and then an algorithm that divides the entry of assignments into sub-streams at prices calculated from the handling capacities of the servers. All these systems are contrasted on two laboratory test beds with homogeneous and heterogeneous subjects with distinct handling capabilities through comparisons between themselves and with a straightforward round-robin scheduler.

DevendraDahiphaleet.al [18]; concentrated on cloud computing and its availability of configurable computing assets attracts a good deal of scrutiny. MapReduce (MR) is a common structure for shared batch job data-intensive computing. Map Reduce has the previous disadvantages: 1 . the handling of Map and Reduce Phases is linear.2. The scalability of the group is comparatively restricted. 3 . It does not encourage elastic sales. 4. It does not promote the handling of information streams. Cloud MapReduce (CMR) is described as overcoming these constraints. Our findings indicate that CMR is more effective and operates quicker than other MR template applications. We also show how CMR can be further upgraded to: 1. Support stream information handling in relation to batch information by using a pipeline model to parallelize the map and reduce stages.2. Support versatile sales using the place cases of Amazon Cloud and cope with huge terminations of machines triggered by changes in place prices. 3. Improve handling throughput and speed-up over traditional MR for big information collections by more than 30 percent. 4 . Provide additional stability and scalability by leveraging the cloud computing design characteristics.

\section{V.SECURITY FOR TASK SCHEDULING IN CLOUD}

LIU Yaqiuet.al [19] concentrated on the safety of task scheduling in the cloud setting and based on the perspective of the problem of multiple Directed Acyclic Graphs (DAGs) planning in the multi-tenant cloud computing setting, a planning approach is suggested that integrates safety and accessibility to meet the material safety and accessibility demands of employees. They focused on resource reputation in order to ensure that jobs can be scheduled on relatively security nodes; during the scheduling of tasks, it classifies the DAGs to achieve fairness; during the resource allocation process, the objective function would maximize the security satisfaction of the user and minimize the availability deviation; meanwhile, it uses "time chips" to promote resource utilization rate; afterwards, they have presented a Greedy algorithm integrating with security and availability (GISA) to implement the strategy. The test findings demonstrate the accuracy and superiority of the new approach. Huangke Chen et.al [20]: concentrated on the broad application of cloud computing in many businesses as well as in science and engineering, where high-quality safety facilities are increasingly critical to the handling of delicate medium information workflow apps. Unfortunately, most current workflow planning methods ignore the optimal data security demands generated by workflows and ignore the efficiency effect of medium data encryption moment on the beginning of subsequent workflow activities. 
In addition, resource inactive time frames, leading from data dependencies among workflow assignments,

were not appropriately utilized to mitigate the effect of data encryption moment on the makespans and financial price of workflows. This article provides a novel task-planning structure for security-sensitive workflows with three novel characteristics to tackle these problems. First, we provide comprehensive theoretical analyzes on how it is helpful to selectively duplicate the predecessor tasks of a task in order to avoid delaying the starting time of both the data transmission time and the encryption time. Then we define the latest completion time of the workflow tasks and prove that tasks can be completed before the latest completion time of the tasks by using the cheapest resources to reduce monetary costs without delaying the start time and the makespans of the workflows of the successors of the tasks. Based on these analyzes, we develop a novel planning strategy with duplication of specific duties, called SOLID, which includes two significant stages:

- Task Scheduling activities in which previous assignments are selectively duplicated to inactive time frames on assets.

- Intermediate information encryption by efficiently exploiting laxity moments of assignments. Using both spontaneously created workflows and some real-world workflow markings, we assess our alternative strategy through strict performance evaluation research. Our findings indicate that the suggested SOLID strategy takes precedence over current algorithms with regard to make-up, financial expenses and energy effectiveness.

\section{VI.METHODOLOGY}

Chien-Hung Chenet.al [21] concentrated on MapReduce, a software framework for the parallel processing of dataintensive apps in cloud computing systems. Some Map Reduce employment have their work execution date demands. The current time-limited MapReduce scheduling systems do not address the previous two issues: different server output and dynamic job efficiency moment. The Bipartite Graph modeling was used in this document to suggest a fresh MapReduce Scheduler called the BGMRS. By transforming the issue into a well-known graph issue, the BGMRS can acquire the optimal answer to the time-limited planning issue: minimum weighted bipartite matching. The previous characteristics are available from the BGMRS. It considers the heterogeneous cloud computing environment, so that some nodes ' computing resources cannot fulfill some job deadlines. In addition to meeting the requirement of the deadline, the BGMRS also includes the data locality in the allocation of the computing resource to shorten a job's data access time. However, if the system's total available computing resources cannot meet all job deadlines, the BGMRS can minimize the amount of employment with the breach of the limit. Finally, both simulation and controlled tests are conducted to show the efficacy of the BGMRS in timely planning.

\section{VII.WORKLOAD ADJUSTMENT FOR HADOOP CLUSTER}

XiaolongXu et.al [22]: suggested that Hadoop's initial task scheduling algorithm could not satisfy heterogeneous cluster efficiency demands. Adaptive Scheduling Strategy based on Dynamic Workload Adjustment (ATSDWA) was provided depending on the continuous shift in demand of each job node and the variation in node results of distinct assignments in the heterogeneous Hadoop group. With ATSDWA, task trackers can adjust to modify the load at runtime, acquire assignments according to their own computing capacity, and carry out self-regulation while reducing the difficulty of the algorithm, which was the main justification to create the service efficiency bot work tracker. Experimental findings have shown that ATSDWA is a highly efficient and reliable algorithm that can stabilize, scalable, effective and load managing heterogeneous Hadoop nodes. In addition, its performance was superior to Hadoop's initial and enhanced task scheduling approach, from the elements of job execution moment, asset usage.

DazhaoChenget.al speed-up ratio [23]: Datacenter-scale nodes have been concentrated on developing towards heterogeneous hardware architectures owing to constant server substitution. Meanwhile, for quite distinct purposes, datacenters are widely distributed by many customers. Because of multi-tenant interferences, it often shows important efficiency heterogeneity. MapReduce implementation on such heterogeneous centers posed important difficulties relative to in-house devoted centers in attaining excellent application performance. Since most MapReduce applications are initially intended for homogeneous settings, heterogeneity, given current optimizations on task scheduling and load balancing, can trigger important decline in job performance. They described in this article that the homogeneous setup of functions on heterogeneous nodes can be a major source of load imbalance and therefore trigger bad efficiency. Tasks should be tailored to suit the capacities of heterogeneous nodes with distinct settings. To this end, they suggested a self-adaptive job tuning strategy that finds the ideal settings for personal assignments operating on distinct nodes automatically. In a heterogeneous group, Ant first splits nodes depending on their hardware settings into a set of homogeneous sub clusters. It then handles each sub-cluster as a separate cluster and the self-tuning matrix relates to them separately. And lastly configures assignments with uniformly chosen settings and progressively improves settings of assignments by reproducing settings from highest performing assignments and discarding bad performance settings. Ant utilizes DNA algorithms during adjustable job setup to improve job adjustment and prevent capturing in local optimum. Experimental findings on a heterogeneous physical group with variable software capacities indicate that Ant improved the median work closure moment by 31 percent, 20 percent, and 14 percent relative to inventory Hadoop (Stock), tailored Hadoop with sector suggestions (Heuristic), and a profiling-based setup strategy (Starfish), respectively. In addition, they expanded Ant in a multitenant private cloud to digital Map Reduce nodes. Specifically, depending on two observed efficiency statistics, Ant characterizes a simulated machine: I / O speed and CPU grab moment. It utilizes the clustering algorithm kmeans to rank virtual nodes depending on the observed vibrant interference into setup classes. Experimental findings on simulated complexes with variable interferences indicate that, opposed to Stock, Heuristic and Starfish, Ant increases the median work finishing moment by 20 percent, 15 percent, and 11 percent.

Blue Eyes Intelligence Engineering

$\&$ Sciences Publication 


\section{VIII.DATA FLOW DIARAM}

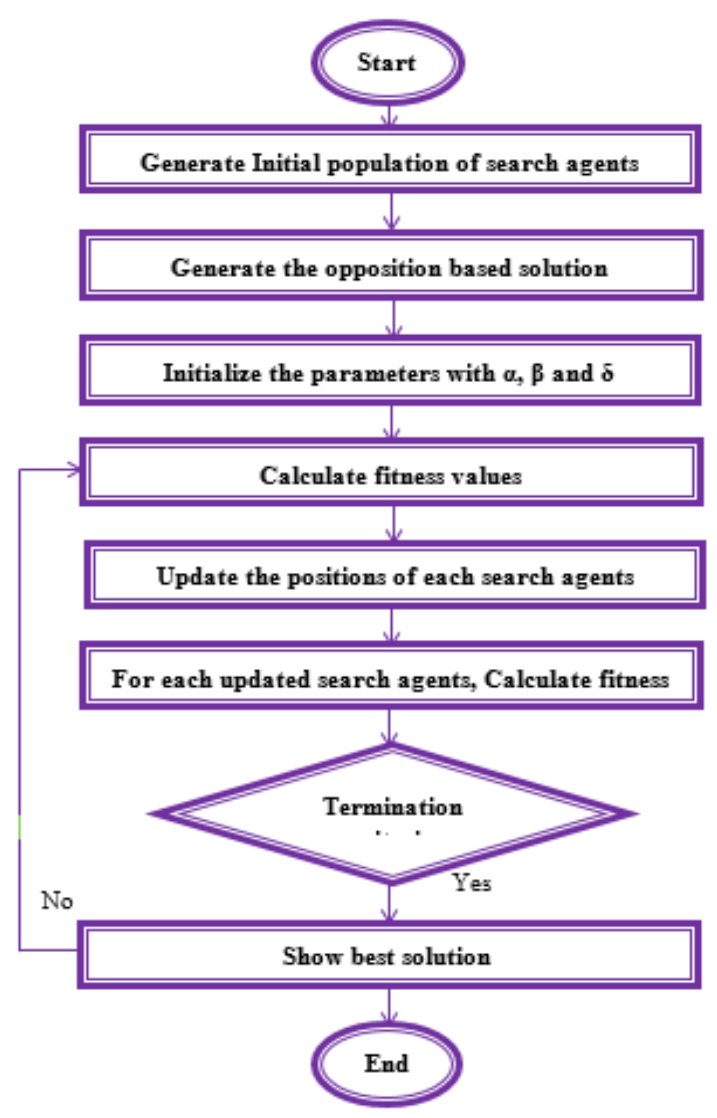

Figure.1: Depicts Data Flow Diagram

The above Fig. 1 contains the working procedure of the proposed method to identify the issues of task scheduling of big data in cloud environment.

\section{IX.TASK ASSIGNMENT USING SPECTRAL CLUSTERING}

Nikolaos D et.al [24]: have been focused on task assignment on cloud and the Resource selection and task assignment are basic operations in distributed computing environments, like the grid and the cloud, where tasks compete for resources. The decisions made by the corresponding algorithms should be judged based not only on metrics related to user satisfaction, such as the percentage of tasks served without violating their quality-of-service (QoS) requirements, but also based on resource-related performance metrics, such as the number of resources used to serve the tasks and their utilization efficiency. In our work, they focused on the case of tasks with fixed but not strict time requirements, given in the form of a requested start and finish time. They proposed an algorithm for assigning tasks to resources that minimizes the violations of the tasks' time requirements while simultaneously maximizing the resources' utilization efficiency for a given number of resources. The exact time scheduling of the tasks on the resources was then decided by taking into account the time constraints. The proposed scheme exploits concepts derived from graph partitioning, and group's together tasks so as to 1) minimize the time overlapping of the tasks assigned to a given resource and 2) maximize the time overlapping among tasks assigned to different resources. The partitioning was performed using a spectral clustering methodology through normalized cuts. Experimental results showed that the proposed algorithm outperforms other scheduling algorithms for different values of the granularity and the load of the task requests.

\section{X.RESULT AND DISCUSSION}

This region shows the relevant consequences of the submitted technique for creating an effective data handling parallelized resource allocation and ideal planning method using Novel algorithm to solve the issue of big data processing in cloud setting. Here we have selected distinct parameters those are Physical Machine, Virtual Machine, Tasks, CPU, Memory and Time to enhance the efficiency of our job. These parameters are implemented to three different problems and their measurement distances are shown in Table-1 below.

Table -1: Task parameters and its range
\begin{tabular}{|c|c|c|c|c|c|c|}
\hline \multicolumn{7}{|c|}{ Range } \\
\hline $\begin{array}{c}\text { Problem/ } \\
\text { Parameter }\end{array}$ & $\begin{array}{c}\text { Physical } \\
\text { Machine }\end{array}$ & $\begin{array}{c}\text { Virtual } \\
\text { Machine }\end{array}$ & Tasks & CPU & Memory & Time \\
\hline P1 & 50 & 126 & 100 & 126 & $1-2 \mathrm{~GB}$ & $100 \mathrm{~ms}$ \\
\hline P2 & 100 & 294 & 200 & 294 & $1-2 \mathrm{~GB}$ & $100 \mathrm{~ms}$ \\
\hline P3 & 200 & 589 & 400 & 589 & $1-2 \mathrm{~GB}$ & $100 \mathrm{~ms}$ \\
\hline
\end{tabular}

Table-1 contains the different parameter values for distinct problems like P1, P2, P3

Table-2: Correlation Matrix

\begin{tabular}{|c|c|c|c|c|}
\hline \multicolumn{5}{|c|}{ Correlation Matrix for Table -1 } \\
\hline & Physical & Virtual & Task & CPU \\
\hline Physical & 1 & 0.9995 & 1 & 0.9995 \\
\hline Virtual & 0.9995 & 1 & 0.9995 & 1 \\
\hline Task & 1 & 0.9995 & 1 & 0.9995 \\
\hline CPU & 0.9995 & 1 & 0.9995 & 1 \\
\hline
\end{tabular}

Table -2 show the relationship exist between different parameters with Correlation Matrix for Table-1

Table-3: PCA Analysis
\begin{tabular}{|c|c|c|}
\hline \multicolumn{2}{|c|}{ PCA Amalysis for Table-1 } \\
\hline Eigen & Variance & Cummlative \\
\hline 3.9989 & 99.9727 & 99.9727 \\
\hline 0.0011 & 0.0273 & 100 \\
\hline 0 & 0 & 100 \\
\hline 0 & 0 & 100 \\
\hline
\end{tabular}

Table-3 shows the Principle Component Analysis with different values for Eigen, Variance, Cumulative related to Table-1.

\section{XI.CONCLUSION}

In this paper, the initial presentation of the concept and history of big data in cloud computing.

Then some preliminaries with the MapReduce programming model and its tastes about big data processing are discussed. This section also discusses the difficulties and problems in handling cloud-based data-intensive apps. The main issue recognized is the planning of data-intensive apps using the MapReduce system in a cloud that is the main goal of this paper widely researched. 


\section{REFERENCES}

1. Zhang, PeiYun, and MengChu Zhou. "Dynamic cloud task scheduling based on a two-stage strategy." IEEE Transactions on Automation Science and Engineering 15, no. 2 (2018): 772-783.

2. Zhong, Zhifeng, Kun Chen, XiaojunZhai, and Shuange Zhou. "Virtual machine-based task scheduling algorithm in a cloud computing environment." Tsinghua Science and Technology21, no. 6 (2016): 660-667.

3. Lin, Chuan, Yuanguo Bi, Guangjie Han, Jucheng Yang, Hai Zhao, and Zheng Liu. "Scheduling for Time-Constrained Big-File Transfer Over Multiple Paths in Cloud Computing." IEEE Transactions on Emerging Topics in Computational Intelligence 2, no. 1 (2018): 2540.

4. Zhao, Yong, Liang Chen, Youfu Li, and WenhongTian. "Efficient task scheduling for Many Task Computing with resource attribute selection." China Communications 11, no. 12 (2014): 125-140.

5. Cheng, Chunling, Jun Li, and Ying Wang. "An energy-saving task scheduling strategy based on vacation queuing theory in cloud computing." Tsinghua Science and Technology 20, no. 1 (2015): 2839.

6. Mastronarde, Nicholas H., and Mihaela van der Schaar. "A queuingtheoretic approach to task scheduling and processor selection for video-decoding applications." IEEE Transactions on multimedia 9, no. 7 (2007): 1493-1507.

7. Yuan, Haitao, Jing Bi, Wei Tan, MengChu Zhou, Bo $\mathrm{Hu} \mathrm{Li}$, and Jianqiang Li. "TTSA: An effective scheduling approach for delay bounded tasks in hybrid clouds." IEEE transactions on cybernetics 47, no. 11 (2017): 3658-3668.

8. Li, Wenxiang, Chunsheng Zhu, Laurence T. Yang, Lei Shu, Edith CH. Ngai, and Yajie Ma. "Subtask scheduling for distributed robots in cloud manufacturing." IEEE Systems Journal 11, no. 2 (2017): 941950.

9. Cui, Hongyan, Yang Li, Xiaofei Liu, Nirwan Ansari, and Yunjie Liu. "Cloud service reliability modelling and optimal task scheduling." IET Communications 11, no. 2 (2017): 161-167.

10. Esteves, Sérgio, and LuísVeiga. "WaaS: Workflow-as-a-Service for the Cloud with Scheduling of Continuous and Data-intensive Workflows." The Computer Journal 59, no. 3 (2016): 371-383.

11. Yuan, Haitao, Jing Bi, MengChu Zhou, and Ahmed ChihebAmmari. "Time-Aware Multi-Application Task Scheduling With Guaranteed Delay Constraints in Green Data Center." IEEE Transactions on Automation Science and Engineering 15, no. 3 (2018): 1138-1151.

12. Zhu, Xiaomin, Laurence T. Yang, Huangke Chen, Ji Wang, Shu Yin, and Xiaocheng Liu. "Real-time tasks oriented energy-aware scheduling in virtualized clouds." IEEE Transactions on Cloud Computing 2, no. 2 (2014): 168-180.

13. Zhang, Qi, Mohamed FatenZhani, Yuke Yang, RaoufBoutaba, and Bernard Wong. "PRISM: fine-grained resource-aware scheduling for MapReduce." IEEE Transactions on Cloud Computing 1 (2015): 1-1.

14. Xhafa, Fatos, and Ajith Abraham. "Computational models and heuristic methods for Grid scheduling problems." Future generation computer systems 26, no. 4 (2010): 608-621.

15. Delimitrou, Christina, and Christos Kozyrakis. "Security implications of data mining in cloud scheduling." IEEE Computer Architecture Letters 15, no. 2 (2016): 109-112.

16. Zuo, Xingquan, Guoxiang Zhang, and Wei Tan. "Self-adaptive learning PSO-based deadline constrained task scheduling for hybrid IaaS cloud." IEEE Transactions on Automation Science and Engineering 11, no. 2 (2014): 564-573.

17. Wang, Lan, and ErolGelenbe. "Adaptive dispatching of tasks in the cloud." IEEE Transactions on Cloud Computing 6, no. 1 (2018): 3345.

18. Dahiphale, Devendra, RutvikKarve, Athanasios V. Vasilakos, Huan Liu, Zhiwei Yu, AmitChhajer, Jianmin Wang, and Chaokun Wang. "An advanced mapreduce: cloud mapreduce, enhancements and applications." IEEE Transactions on Network and Service Management 11, no. 1 (2014): 101-115.

19. Liu, Yaqiu, Hongrun Shao, Weipeng Jing, and ZhaowenQiu. "MultiDAGs scheduling integrating with security and availability in cloud environment." Chinese Journal of Electronics 24, no. 4 (2015): 709716.

20. Chen, Huangke, Xiaomin Zhu, DishanQiu, Ling Liu, and Zhihui Du. "Scheduling for workflows with security-sensitive intermediate data by selective tasks duplication in clouds." IEEE Transactions on Parallel and Distributed Systems 28, no. 9 (2017): 2674-2688.

21. Chen, Chien-Hung, Jenn-Wei Lin, and Sy-Yen Kuo. "MapReduce scheduling for deadline-constrained jobs in heterogeneous cloud computing systems." IEEE Transactions on Cloud Computing 6, no. 1 (2018): 127-140.

22. Dr. Chetana Tukkoji et.al"ITM-CLD: Intelligent traffic management to handling cloudlets of the large data"CSOC 2018: Cybernetics and Algorithms in intelligent Systems, $17^{\text {th }}$ May 2018. Part of Advanes in Intelligent Systems and Computing, book series AISC volume765

23. $\mathrm{Xu}$, Xiaolong, Lingling $\mathrm{Cao}$, and Xinheng Wang. "Adaptive task scheduling strategy based on dynamic workload adjustment for heterogeneous Hadoop clusters." IEEE Systems Journal 10, no. 2 (2016): 471-482.

24. Cheng, Dazhao, JiaRao, YanfeiGuo, Changjun Jiang, and Xiaobo Zhou. "Improving performance of heterogeneous mapreduce clusters with adaptive task tuning." IEEE Transactions on Parallel and Distributed Systems 28, no. 3 (2017): 774-786.

25. Doulamis, Nikolaos D., Panagiotis Kokkinos, and EmmanouelVarvarigos. "Resource selection for tasks with time requirements using spectral clustering." IEEE Transactions on Computers 63, no. 2 (2014): 461-474.

\section{AUTHORS PROFILE}

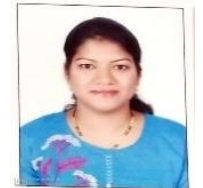

Dr. Chetana Tukkoji, obtained her Bachelor's degree in Computer Science and Engineering from Visvesvaraya Technological University, KLECET, Belagavi in 2009. Then she obtained her Master's degree in Computer Science and Engineering from BVBCET, Hubli in 2012 and Completed PhD in Cloud ComputingBigData Domain, from Visvesvaraya Technological University in 2019 Currently, she is working as an Assistant Professor in the Department of Computer Science and Technology, School of Technology, GITAM Deemed to be University, Bangalore. Her areas of interest include Cloud computing, Computer network, Data mining, Robotic Process Automation and Artificial Intelligence. She is having 8 years of teaching and one year of industry experience. She has also published 15 journals including national/international and two book books online on C Programming for Beginners and Computer Organizations and Architecture.

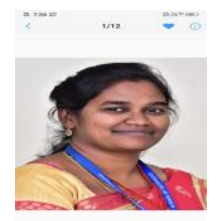

Mrs. Boosi Shyamala, obtained her Bachelor's degree in Computer Science and Engineering from Jawaharla Nehru Technological University, SICET, Rangareddy in 2005. Then she obtained her Master's degree in Computer Science and Engineering from BVRIT, Medak in 2012 and Pursuing PhD in Artificial Intelligence and Machine Learning Domain, in GITAM deemed to be University from 2018. Currently, she is working as an Assistant Professor in the Department of Computer Science and Technology, School of Technology, GITAM Deemed to be University, Bangalore. Her area of interest includes Artificial Intelligence, Machine Learning, Robotic Process Automation, Data mining and Big Data Analytics. She has 13 years of teaching and one year of industrial experience. She has 8 publications in national/ international journals. She had qualified as an Assistant professor UGC-NET, in December 2018. She has successfully completed a DST (Department of Science and Technology) funded project.

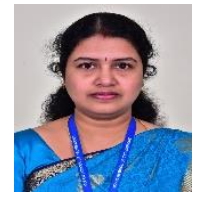

Mrs. Archana S Nadhan, is an assistant professor in the department of computer science and engineering, School of Technology, GITAM Deemed to be University, Bengaluru Campus. She has 13 years of teaching experience and currently she is pursuing $\mathrm{PhD}$. in IoT security in GITAM deemed to be University from 2017. Her areas of interest include Computer networks, IoT and cyber security.

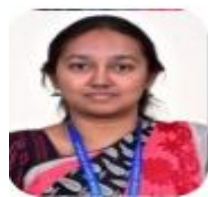

Ms Rashmi K., obtained her Bachelor's degree in Computer Science and Engineering from Visvesvaraya Technological University, RLJIT, Doddaballapur in 2011. Then she obtained her Master's degree in computer networks from CMRIT, Bengaluru in 2015. Currently she is working as assistant Professor in Department of CS\&E, GITAM School of Technology, Bengaluru Campus. She Published a book related to computer science on Computer Networks (ISSBN: 9789386770707) by Aram Book House (P) Ltd in 2018.The research interests are Computer networks, Artificial intelligence and wireless sensors networks. She is having 4 years in teaching experience. She has also published 10 journals including national/ international. 